\title{
Superconducting materials and applications*
}

\author{
T S RADHAKRISHNAN \\ Indira Gandhi Centre for Atomic Research, Kalpakkam 603 102, India
}

\begin{abstract}
The field of superconductivity has witnessed tremendous excitement in recent years, starting with the discovery of what has come to be known as 'high temperature superconductors'. This has led to extensive activity on many aspects concerning the mechanism of superconductivity, new materials and systems and their technological applications. Further impetus to research in this area has been provided by the discovery of superconductivity in doped fullerenes. The first identification of superconductivity in a quarternary borocarbide system $\mathrm{Y}-\mathrm{Ni}-\mathrm{B}-\mathrm{C}$ which must be regarded as the foremost fundamental Indian contribution in recent times, has further stimulated interest in this field. Notwithstanding the new excitements, the conventional superconductors continue to be the workhorses for technological applications. This review selectively presents some of the aspects of the developments in the entire gamut of the known superconducting systems from the stand point of materials and their applications.
\end{abstract}

Keywords. Superconducting materials; $\mathrm{Nb}-\mathrm{Ti}$; HTSC; fullerenes; borocarbides; applications.

\section{Introduction}

\subsection{Recent advances in superconductivity}

The field of superconductivity has witnessed tumultous advances in the last decade starting with the discovery of the 'high temperature superconductors'(HTSC)(Bednorz and Muller 1986) in the system $\mathrm{La}-\mathrm{Ba}-\mathrm{Cu}-\mathrm{O}$ with a transition temperature $\left(T_{\mathrm{c}}\right)$ of $35 \mathrm{~K}$. This was quickly followed up with the identification of superconductivity in several new materials belonging to the class of layered perovskite cuprates with $T_{\mathrm{c}}$ as high as $125 \mathrm{~K}$ (for a comprehensive list, see Radhakrishnan et al 1989a. Since then $\mathrm{Hg}-\mathrm{Ba}-\mathrm{Ca}-\mathrm{Cu}-\mathrm{O}$ has been added to the list with a $T_{\mathrm{c}}$ of $135 \mathrm{~K}$ at $1 \mathrm{~atm}$.). There are reports of highly metastable cuprate structures produced by layer by layer deposition techniques giving indications of superconductivity, although unverified, at $180 \mathrm{~K}$ and even at $250 \mathrm{~K}$ (Locquet and Machler 1994). The goal here clearly is the Holy Grail of room temperature superconductivity. Another significant advance in a totally different direction is the discovery of superconductivity (Hebard et al 1991) in potassium doped solid $\mathrm{C}_{60}$, a new form of carbon which in itself is an insulator. Following this, superconductivity has been observed in other alkali doped $\mathrm{C}_{60}$ systems with the maximum $T_{\mathrm{c}}$ being $33 \mathrm{~K}$ in $\mathrm{Rb}_{2} \mathrm{CsC}_{60}$. Yet another and the latest in the series of exciting advances is the discovery of superconductivity in $\mathrm{Y}-\mathrm{Ni}-\mathrm{B}-\mathrm{C}$ at $12-15 \mathrm{~K}$ (Nagarajan et al 1994). This has subsequently opened up the class of quarternary borocarbide superconductors with the highest $T_{\mathrm{c}}$ so far known of $23 \mathrm{~K}$ in $\mathrm{Y}-\mathrm{Pd}-\mathrm{B}-\mathrm{C}$ system, a record for bulk intermetallics. All these advances have triggered new levels of activity in the science and technology of superconducting materials and many interesting properties of these classes of superconductors have come to light.

*An update of the article "Current trends in the development and applications of superconducting materials", Sundaram and Radhakrishnan (1989) 
Table 1. Important classes of superconductors of contemporary interest.

\begin{tabular}{|c|c|c|c|}
\hline Superconductor & $\begin{array}{l}T_{\mathrm{c}} \mathrm{Max} \\
\quad(\mathrm{K})\end{array}$ & $\begin{array}{c}H_{\mathrm{c} 2} \\
(\mathrm{Tesla} ; 4 \cdot 2 \mathrm{~K})\end{array}$ & Major interest \\
\hline Niobium-titanium & 11 & 12 & Most used in applications \\
\hline $\mathrm{A} 15\left(\right.$ e.g. $\left.\mathrm{Nb}_{3} \mathrm{Sn}\right)$ & 23 & 40 & $\begin{array}{l}\text { Used in applications beyond } \\
8 \text { Tesla }\end{array}$ \\
\hline $\begin{array}{l}\text { Ternary molybdenum } \\
\text { chalcogenides } \\
\left(\text { e.g. } \mathrm{PbMo}_{6} \mathrm{~S}_{8}\right)\end{array}$ & 14 & 60 & $\begin{array}{l}\text { Interest in the context of co- } \\
\text { existence of superconductivity } \\
\text { and magnetism; possible appli- } \\
\text { cation in high fields }\end{array}$ \\
\hline $\begin{array}{l}\text { High } T_{\mathrm{c}} \text { superconductors } \\
\text { (e.g. } \mathrm{YBa}_{2} \mathrm{Cu}_{3} \mathrm{O}_{7} \text { ) }\end{array}$ & 135 & 100 & $\begin{array}{l}\text { Potential for applications at } \\
77 \mathrm{~K} ; \mathrm{H}-\mathrm{T} \text { phase diagram; } \\
\text { irreversibility line; mechanism } \\
\text { of superconductivity }\end{array}$ \\
\hline Doped fullerenes (e.g. $\mathrm{K}_{3} \mathrm{C}_{60}$ ) & $31 \cdot 3$ & & Mechanism of superconductivity \\
\hline $\begin{array}{l}\text { Quarternary borocarbides } \\
\left(\text { e.g. } \mathrm{YNi}_{2} \mathrm{~B}_{2} \mathrm{C}\right)\end{array}$ & 23 & & $\begin{array}{l}\text { Coexistence of magnetism and } \\
\text { superconductivity }\end{array}$ \\
\hline
\end{tabular}

\subsection{Early phase-conventional superconductors}

Prior to these spectacular advances, binary intermetallics had been the favourite hunting ground for researchers in superconductivity. Although the goal had been to increase the $T_{\mathrm{c}}$ as high as possible, the efforts succeeded in pushing it up only to about $23 \mathrm{~K}$. Nevertheless, nearly three decades of materials research since the 1957 theoretical proposal of type II superconductivity by Abrikosov saw three outstanding classes of superconductors being developed. These are the niobium-titanium ( $\mathrm{Nb}-\mathrm{Ti})$ solid solution system, the $\mathrm{A} 15$ superconductors (e.g. $\mathrm{Nb}_{3} \mathrm{Sn}, \mathrm{V}_{3} \mathrm{Ga}$ ) and the chevrel phase superconductors (CPC--e.g. $\mathrm{PbMo}_{6} \mathrm{~S}_{8}$ ). These are sometimes called conventional or the low temperature superconductors (LTSC) to distinguish them from the HTSC referred to earlier. The important classes of conventional superconductors along with those of contemporary interest are listed in table 1 with their basic properties.

\subsection{Superconductors in science and technology}

Superconducting materials are generally looked at from two view points. One is their interest in science, primarily with respect to the mechanism of pairing, whether they can be described by the standard BCS type theory with electron-phonon coupling or whether strong electron correlations are operative. The other interest is the assessment of their usefulness in technology as to whether they have the requisite properties and are capable of being cast into forms suitable for applications. The author had occasion (Sundaram and Radhakrishnan 1989) to review the then trends in the development and applications of superconducting materials. This will henceforth be referred as review I and will be cited where necessary. Therein a comprehensive introduction to superconductivity, the basis for applications in technology and properties of relevance to such applications have been discussed. Review I also discussed extensively the three most important classes of LTSC in vogue then viz. the $\mathrm{Nb}-\mathrm{Ti}$, the A15 and the CPC. This 
review will trace the advances since then both in terms of new discoveries and also from the point of view of applications. Of the materials listed in table 1 , the $\mathrm{Nb}-\mathrm{Ti}$, the CPC and the HTSC will be discussed more elaborately in view of their importance as technological materials. In contrast to HTSC, doped fullerenes and the quarternary borocarbides do not seem to have great technological potential as superconductors and they will be mentioned only briefly.

\section{Niobium-titanium}

\subsection{Development of high current conductors}

The basic technological issues concerning the $\mathrm{Nb}-\mathrm{Ti}$ have been discussed in review $\mathrm{I}$. The alloys of commercial interest lie in the compositional range $\mathrm{Nb}-40$ to $65 \mathrm{wt} \% \mathrm{Ti}(\mathrm{Nb}-55$ to 78 at $\%$ Ti). Despite the progress made in the study of HTSC, Nb-Ti alloys still remain the most used superconductors for high current applications. Review I mentioned that the best laboratory scale conductors developed by 1986 had a critical current density $J_{c}$ of $3500 \mathrm{~A} / \mathrm{mm}^{2}(5 \mathrm{~T} ; 4 \cdot 2 \mathrm{~K})$ and those in production, $2700 \mathrm{~A} / \mathrm{mm}^{2}$. This situation was largely due to the fillip given by the plans to set up the superconducting supercollider (SSC) in the US, which has since been given up. Mainly, increase in $J_{c}$ was achieved by improvement in the alloy homogeneity at the ingot stage and multiple step heat treatment schedules during wire drawing for the formation of $\alpha$-Ti precipitates as flux pinning centres. A significant advance has been the realization of multifilamentary conductors with fine filaments down to $1 \mu \mathrm{m}$ and below, which eliminated the need for individual correction coils on the bore tube of dipole magnets to achieve field homogeneity. An acute problem in this was the formation of $\mathrm{Cu}_{3} \mathrm{Ti}$ precipitates which were brittle and which caused reduction in filament diameters called 'sausaging'. This is prevented by introducing a suitable diffusion barrier around the filaments during fabrication.

Apart from these extrinsic factors, intrinsic factors related to the microstructural features controlling $J_{\mathfrak{c}}$ were identified. It was found that high $J_{\mathfrak{c}}$ can be achieved by developing a fine defect structure consisting of elongated ribbons of $\alpha$-Ti precipitates and sub-grain boundaries. The size, shape and separation of these pinning defects could be optimized by a judicious selection of cold work and heat treatment. In several conductors with $J_{\mathrm{c}}$ ranging from $1500 \mathrm{~A} / \mathrm{mm}^{2}$ to $3500 \mathrm{~A} / \mathrm{mm}^{2}$ ( 5 Tesla; $4 \cdot 2 \mathrm{~K}$ ), it has been found that $J_{\mathrm{c}}$ when plotted against the true strain (from the hot extended size to the final wire size), bears a remarkable linear relationship provided $J_{\mathrm{c}}$ is not limited by extrinsic factors like sausaging. Extrapolation indicates that a $J_{c}$ of $4000 \mathrm{~A} / \mathrm{mm}^{2}$ may be possible by extending the total strain in the range of 14 or 15 . A further development has been the use of artificial pinning centres in the processing of $\mathrm{Nb}-\mathrm{Ti}$ alloy. Using pure $\mathrm{Nb}$ as pinning material, conductors have been developed with a record low field $J_{\mathrm{c}}$ of $7500 \mathrm{~A} / \mathrm{mm}^{2}$ at $3 \mathrm{~T}$ on laboratory samples (Kanithi et al 1992). This is twice that of the present conductors used for magnetic resonance imaging systems. Further, a $J_{\mathrm{c}}$ of $3400 \mathrm{~A} / \mathrm{mm}^{2}$ at $5 \mathrm{~T}$ has been obtained by this method.

\subsection{Lattice instability and structural transformation}

The $\mathrm{Nb}-\mathrm{Ti}$ alloys in the compositional range of commercial interest are metastable when quenched from the high temperature $\beta$ (bcc) phase. On further cooling, either 
there can be an incipient lattice instability or there could be an occurrence of a structural transformation. The structural transformation if occurs can be by one of two modes, either a martensitic transformation or the formation of hexagonal athermal $\omega$ precipitates. The occurrence of the one or the other mode depends upon the concentration of $\mathrm{Ti}$, quench rate, temperature and stress.

Many physical properties of $\mathrm{Nb}-\mathrm{Ti}$ show anomalies which have been well documented (Collings 1983; Radhakrishnan 1987). For example the normal state resistivity increases as Ti concentration increases but decreases markedly for 83 at $\% \mathrm{Ti}$, signifying a structural transformation. In one investigation, this alloy could be retained (Hariharan et al 1986a, b) in the metastable $\beta$ phase by the addition of 1 at $\%$ nitrogen, introduced by an ion nitriding process. Consequently the resistivity increased to a very high value of $150 \mu \Omega \mathrm{cm}^{-1}$, the largest known for $\mathrm{Nb}-\mathrm{Ti}$ alloys. Similar anomaly was also found in $\mathrm{d} \rho / \mathrm{d} T$. In another investigation (Obst et al 1980) the electrical resistivity of a quenched $\mathrm{Nb}-78$ at $\%$ alloy exhibited predominantly negative temperature coefficient of resistance in the temperature region above $100 \mathrm{~K}$. On application of stress at $180 \mathrm{~K}$ the value of resistivity decreased and $\mathrm{d} \rho / \mathrm{d} T$ became positive. The results indicate that the lattice instability is relieved by a structural transformation due to application of stress.

The effect of lattice instability on the superconducting properties have not been well investigated, apart from the measurements of $T_{\mathrm{c}}$. It is generally believed that the relatively higher $T_{\mathrm{c}}$ exhibited by the quenched alloys compared to the cold worked alloys is a result of the instability in the former. To investigate the effect of the instability on the phonon modes, it is desirable to carry out superconducting tunneling studies. As a first step several alloy films have been made by RF sputtering in the composition range 60 to 90 at \% Ti (Awadhesh et al 1996). The electrical properties shown in figure 1 exhibit anomalies similar to those of the bulk, characteristic of $\beta$ instability. It is

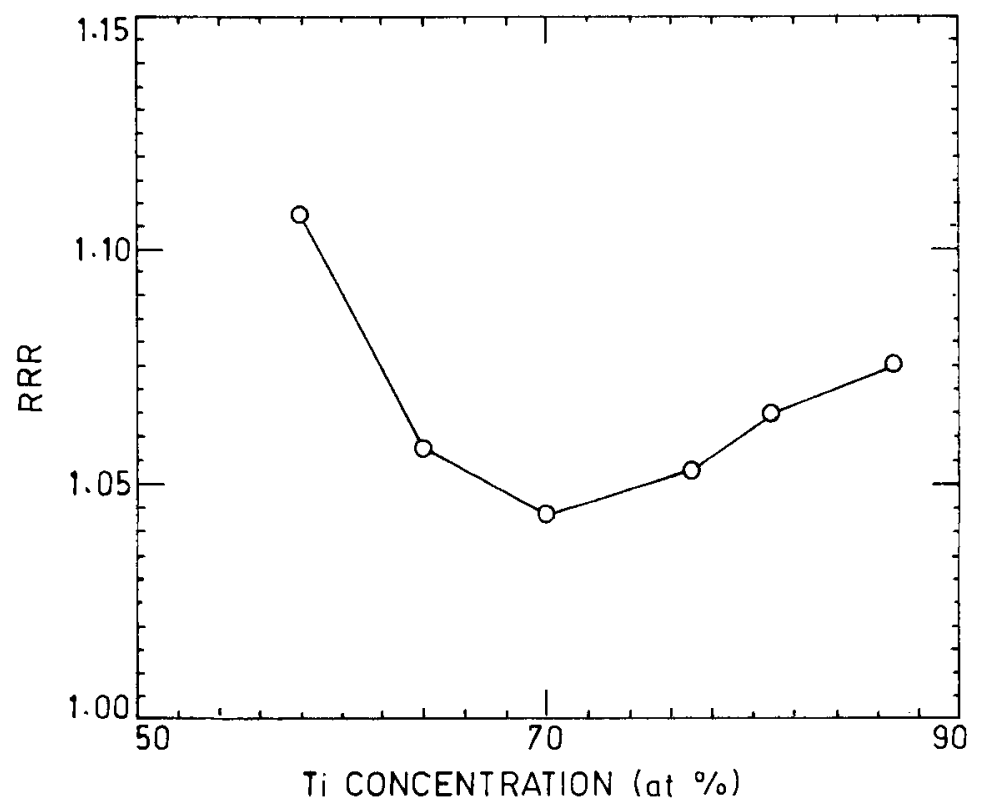

Figure 1. Variation in the electrical property-residual resistivity ratio (RRR) as a function of Ti concentration. 
interpreted that for compositions below $70 \mathrm{at} \% \mathrm{Ti}$, the incipient instability is retained and beyond this composition, it is relieved. Tunneling investigations with the $\mathrm{Nb}-\mathrm{Ti}$ film as one of the electrodes may give valuable information.

\section{Ternary molybdenum chalcogenides $-\mathrm{PbMo}_{6} \mathrm{~S}_{8}$}

\subsection{Development of conductors}

$\mathrm{PbMo}_{6} \mathrm{~S}_{8}$ is the most investigated superconductor in the family of ternary molybdenum chalcogenides with the general formula $\mathrm{M}_{x} \mathrm{Mo}_{6} \mathrm{X}_{8}(\mathrm{M}$-metal; $\mathrm{X}$--chalcogen; $x \sim 1$ ) which are also known as the chevrel phase compounds (CPC). The structure has an overall rhombohedral symmetry and is made up of cubes formed by sulphur (chalcogen) atoms, each containing an Mo octahedral cluster. The metal atom e.g. $\mathrm{Pb}$, is located in the channels running along the rhombohedral axes. Among the many CPC known, $\mathrm{PbMo}_{6} \mathrm{~S}_{8}, \mathrm{Gd}_{0.8} \mathrm{Mo}_{6} \mathrm{~S}_{8}$ and $\mathrm{SnMo}_{6} \mathrm{~S}_{8}$ have been thought of as potential candidates for applications on account of their high $H_{c 2}$ (in the range 40-60 Tesla). Before the advent of $\mathrm{HTSC}, \mathrm{PbMo}_{6} \mathrm{~S}_{8}$ received a lot of attention for development for application in the high field regime beyond 20 Tesla.

$\mathrm{PbMo}_{6} \mathrm{~S}_{8}$ is brittle and a support material has to be used to draw it in the wire form by cold extrusion. During extrusion, the material fractures and to promote electrical connectivity between the fractured surfaces, sintering (recovery anneal) has to be carried out at about $900^{\circ} \mathrm{C}$. CPC being very reactive, this process could result in deleterious effects on $T_{\mathrm{c}}$ and $H_{\mathrm{c} 2}$. This problem is to a certain extent overcome by interposing barrier materials like $\mathrm{Nb}, \mathrm{Mo}, \mathrm{Ag}$ and $\mathrm{Ta}$, which are non-reactive in the sense of formation of second phases and also ductile at room temperature. In addition, apart from the mandatory copper sheath for stabilization against flux jumps, a reinforcing outer layer of stainless steel has to be provided for withstanding the Lorentz forces encountered in high magnetic field applications. The different components of wire have to be matched for thermal expansion, both during the recovery annealing heat treatment (usually at $900^{\circ} \mathrm{C}$ ) and during subsequent cooling in use. The effects of possible stresses due to differential thermal expansion of the different components, on the superconducting properties have not been fully analysed.

There have been many efforts at development of the $\mathrm{PbMo}_{6} \mathrm{~S}_{8}$ wire with high $J_{\mathrm{c}}$. Hirrien (1986) using unreacted mixtures of $\mathrm{PbS}, \mathrm{Mo}_{2} \mathrm{~S}_{3}$ and $\mathrm{Mo}$ and with $\mathrm{Nb}$ barrier obtained a final $J_{c}$ of $100 \mathrm{~A} / \mathrm{mm}^{2}$ at 20 Tesla. Goldacker et al (1988) using hot isostatic pressing (HIP) for achieving high densification of the $\mathrm{PbMo}_{6} \mathrm{~S}_{8}$, have developed monofilamentary wires that showed a $J_{c}$ of $200 \mathrm{~A} / \mathrm{mm}^{2}$ at 20 Tesla. They used Ta barrier and $\mathrm{PbMO}_{6} \mathrm{~S}_{8}$ was pre-reacted before drawing and recovery anneal. These values though small are still significant considering that they are at very high fields. Nevertheless, $\mathrm{PbMo}_{6} \mathrm{~S}_{8}$ still remains only a potential material requiring further development.

At a recent international workshop (Fisher et al 1991) on chevrel phase superconductors, it was concluded that microstructural control, granularity and increasing $J_{c}$ are issues that lie at the heart of the applications of the chevrel phases. It was emphasized that the high $H_{\mathrm{c} 2}$ and short coherence length of $\mathrm{PbMo}_{6} \mathrm{~S}_{8}$ might produce granular effects. But experiments on hot isostatically pressed samples did not show any effect of granularity, which may not be an intrinsic property. Extensive studies of flux 
pinning do not exist and sources of flux pinning are not proven. The inverse grain size dependence on $J_{\mathrm{c}}$ found in some cases suggests that grain boundaries are the dominant pinning centres. The large variation in superconducting properties found for chevrel phases was due to substitutional oxygen impurities. Oxygen could substitute for axial sulphur atoms with the resultant displacement of $0.8 \AA$ of the metal ion towards the impurity oxygen atom.

\subsection{Structural transformation and superconductivity in CPC with divalent metal ions}

In CPC having divalent metal ions, it is known that the lattice is unstable at low temperatures and undergoes a rhombohedral to triclinic distortion (Baillif et al 1981; Geetha et al 1984). The high temperature rhombohedral phase is favourable for superconductivity and if it is retained on cooling, the material becomes a superconductor (e.g. $\mathrm{PbMo}_{6} \mathrm{~S}_{8}$ ). If in the process a structural transformation to triclinic phase intervenes, a gap in the excitation spectrum opens at the Fermi surface and the material behaves as a semiconductor (e.g. EuMo ${ }_{6} \mathrm{~S}_{8}$-Janawadkar et al 1984). This feature appears to be a general behaviour of all divalent CPC. X-ray diffraction studies have shown that the structural transformation to triclinic phase takes place for $E \mathrm{Mo}_{6} \mathrm{~S}_{8}$ below $100 \mathrm{~K}$ and for $\mathrm{BaMo}_{6} \mathrm{~S}_{8}$ below about $180 \mathrm{~K}$. Recently using high resolution neutron diffraction data it was shown (Jorgensen and Hinks 1985) that even the systems $\mathrm{PbMo}_{6} \mathrm{~S}_{8}$ and $\mathrm{SnMo}_{6} \mathrm{~S}_{8}$, which spontaneously become superconducting on cooling, do indeed show small structural distortions from the rhombohedral phase below about $110 \mathrm{~K}$ and $130 \mathrm{~K}$ respectively. At the superconducting temperatures (below $15 \mathrm{~K}$ ) these materials exist as mixed rhombohedral and triclinic phases (Yao et al 1988). As we know that the triclinic phase is nonsuperconducting, the existence of mixed phase is bound to have deleterious effects on superconductivity. It must be mentioned here that no systematic study exists on the influence of such two-phase behaviour on $J_{\mathrm{c}}$ for $\mathrm{PbMo}_{6} \mathrm{~S}_{8}$.

The structural transformation in CPC, when it occurs, is rapidly suppressed by two factors. One is the application of external pressure and the other is alloying to form pseudo-ternary systems. In both cases the structural transformation temperature is rapidly decreased and eventually superconductivity appeared. Much studied (Janawadkar et al 1984) system in this connection is $\mathrm{EuMo}_{6} \mathrm{~S}_{8}$, which as was mentioned earlier is semiconducting at low temperatures and ambient pressure.

\section{High temperature superconductors}

In nearly a decade of preoccupation with the high temperature superconductors, advances have been made on several issues related to the mechanism of superconductivity, as well as the material properties on the one hand and those related to their applications for use on the other. Several topics have been in the forefront in the former category. Discussion of the symmetry of the wave function and flux lattice stability are among those that have been reviewed extensively (Beasley 1995; Zeldov et al 1995). From the materials point of view YBCO has been the most studied and the configurations of oxygen atoms in the basal plane has been of interest with regard to the structure and superconductivity. This topic will be reviewed particularly in the context of work carried out by the author. The discussion related to the developments on application of HTSC will be provided in a separate section on superconductor applications. 


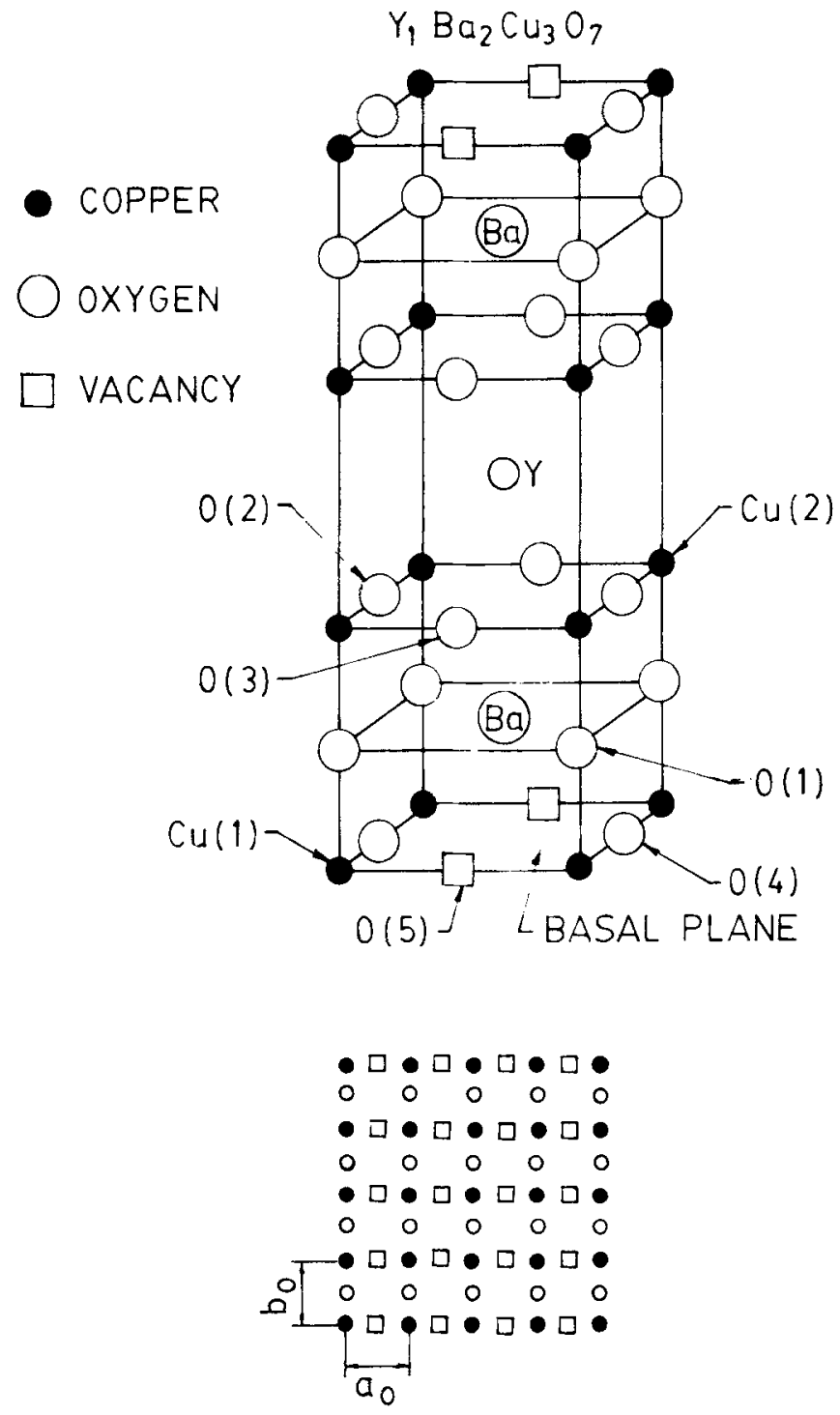

Figure 2. Crystal structure of $\mathrm{YBa}_{2} \mathrm{Cu}_{3} \mathrm{O}_{7} \cdot \mathrm{O}(4)-\mathrm{Cu}-\mathrm{O}(4)$ chain ordering is energetically favourable. For non-stoichiometric samples, several ordered configurations may be realized by the formation of intact and empty chains with respect to oxygen. Bottom: Basal plane showing $\mathrm{Cu}(1)-\mathrm{O}(4)$ chains and oxygen structural vacancies.

\subsection{Oxygen ordering in $\mathrm{YBa}_{2} \mathrm{Cu}_{3} \mathrm{O}_{7-x}$}

The superconducting and normal state properties of $\mathrm{YBa}_{2} \mathrm{Cu}_{3} \mathrm{O}_{7-x}(\mathrm{YBCO})$ depend both on the value of the oxygen stoichiometry and the way these oxygen atoms are arranged in the basal plane of the structure (figure 2). It is now amply clear that the removal or addition of oxygen atoms, as the stoichiometry changes from $\mathrm{O}_{6}$ to $\mathrm{O}_{7}$, takes place only in the basal plane of the structure. Due to the $\mathrm{O}-\mathrm{O}$ attractive interaction mediated by copper, $\mathrm{O}-\mathrm{Cu}-\mathrm{O}$ chain formation is energetically favoured, as 
this together with the nature of interaction between filled and empty chains lead to a variety of ordered configurations in these systems. Depending on the details of sample preparation one can have intra and inter chain disorder and also disorder arising due to random occupation of the $O(4)$ and $O(5)$ sites leading to the orthorhombic to tetragonal transformation.

Oxygen ordering studies carried out fall into three broad categories. In the first type of experiments, various oxygen stoichiometries and order are realized by varying the annealing temperature and the partial pressure of oxygen. The latter determines the degree of order or disorder between $O(4)$ and $O(5)$ sites. Samples annealed in pure oxygen atmosphere exhibit lower room temperature resistivity, sharper superconducting transitions and sharper reflections in XRD compared to those annealed in air, for the same value of $x$ (Hariharan et al 1988; Sankara Sastry et al 1988). A higher resistivity consistent with larger disorder correlates with broader superconducting transitions. A sharp discontinuous change in positron lifetime is also observed at the $\mathrm{T}-\mathrm{O}$ transition, signifying the suppression of $\mathrm{O}(4)-\mathrm{O}(5)$ disorder.

In the second class of studies, oxygen deficient samples are prepared taking care to see that no $\mathrm{O}(4)-\mathrm{O}(5)$ disorder exists. The oxygen vacancies thus go to disrupt the chains in a disordered manner. This being energetically unfavourable, the samples disproportionate into regions having completely filled chains (orthorhombic structure) and regions with empty chains (tetragonal structure) as exemplified upon ageing at $200^{\circ} \mathrm{C}$. The kinetics of this phase separation depends on the annealing environment (like air, oxygen, helium etc). This picture is consistent with the analysis of the results of room temperature resistivity, superconducting transition, XRD, infrared absorption, positron annihilation and oxygen inventory (Sood et al 1988; Radhakrishnan et al 1989a). In a specific study (Radhakrishnan et al 1989b), a sample having oxygen stoichiometry 6.78 (orthorhombic) phase separated into an $O$ phase $(85 \%$ ) with an oxygen stoichiometry 6.92 and a $T$ phase $(15 \%)$ having a stoichiometry $6 \cdot 15$. The sample showed a sharper and higher superconducting transition (figure 3), characteris-

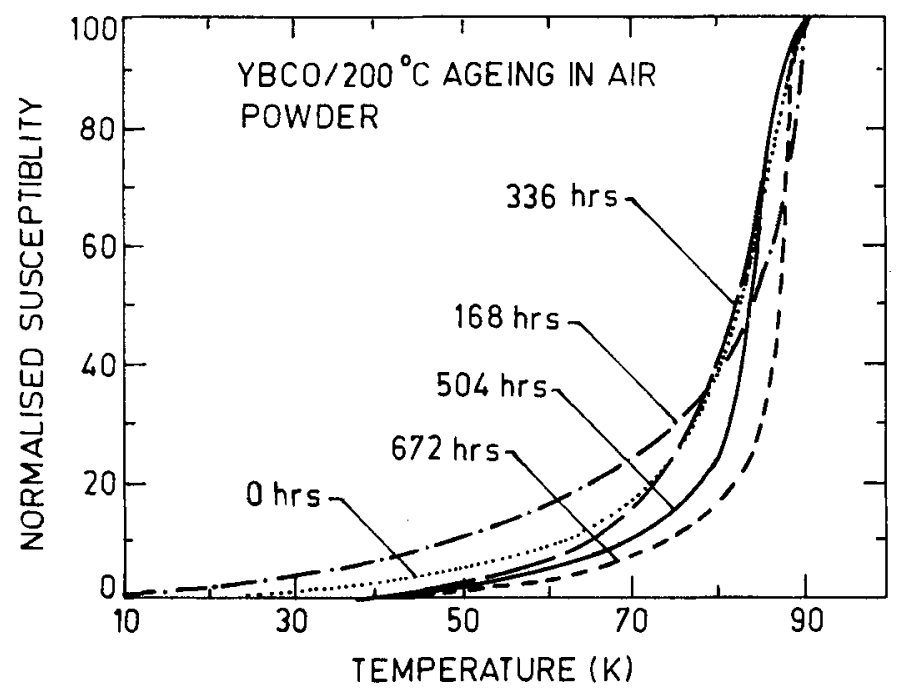

Figure 3. Superconducting transitions in a YBCO sample with oxygen stoichiometry 6.78 on ageing at $200^{\circ} \mathrm{C}$ in air. Higher $T_{\mathfrak{c}}$ and sharpening of the transition is due to phase separation with one phase richer in oxygen content. 
tic of the phase in which oxygen stoichiometry is enriched. These results have been qualitatively understood on the basis of proximity induced influence on the $T_{\mathrm{c}}$ of host by non superconducting $\mathrm{T}$ phases (Baskaran et al 1988).

In the third class of experiments, well ordered oxygen deficient samples in the stoichiometry range 6.78 to 6.36 are prepared by isocompositional slow cooling routines, and are investigated by SAXS and conventional powder diffraction methods. The patterns up to $2 \theta=20^{\circ}$ could be indexed as characteristic of a superstructure with $a^{\prime}=4 a, b^{\prime}=b$ and $c^{\prime}=4 c$, where $a, b, c$ are the lattice parameters of the original YBCO structure. This is the first evidence for superstructure in YBCO by conventional powder XRD measurements. Other measurements have relied heavily on TEM observed on very small regions.

To understand the conditions for obtaining macroscopic regions of ordered domains in situ electrical resistance measurements and in situ high temperature XRD (HTXRD) studies at high temperatures have been carried out in samples subjected to specified heat treatments (Rao et al 1996). Phase pure YBCO with oxygen content, $x=6.9$ were subjected to a typical temperature profile (heating the sample at $50^{\circ} \mathrm{C} / \mathrm{h}$ to reach a desired annealing temperature $\left(T_{\mathrm{A}}\right)$, soaking at $T_{\mathrm{A}}$ for $24 \mathrm{~h}$ followed by slow cooling it at the rate of $13^{\circ} \mathrm{C} / \mathrm{h}$ ). $R$ vs $T$ plots for, $T_{\mathrm{A}}=383^{\circ}, 433^{\circ}$ and $453^{\circ} \mathrm{C}$ are shown in figure 4. The results show several interesting features: (i) increase of resistance in the heating cycle, which may be predominantly due to loss of oxygen, (ii) increase in resistance during soaking at $T_{\mathrm{A}}$, which may be mainly due to the transition from order to disorder in the oxygen configuration and (iii) fall of resistance in the cooling cycle during cooling. While the $383^{\circ} \mathrm{C}$ sample has a linear fall of $R$ with $T$, the $433^{\circ} \mathrm{C}$ and $453^{\circ} \mathrm{C}$ samples show non-linear variation. XRD measurements showed that at the end of the temperature profiles, the samples were orthorhombic with oxygen contents being 6.55 , 6.34 and 6.25 , as estimated from the lattice parameter measurements. For $433^{\circ} \mathrm{C}$ and $453^{\circ} \mathrm{C}$ samples the minima in $\mathrm{d} R / \mathrm{d} T$ curve may be identified to be the temperature at which the samples should be annealed to promote optimal ordering.

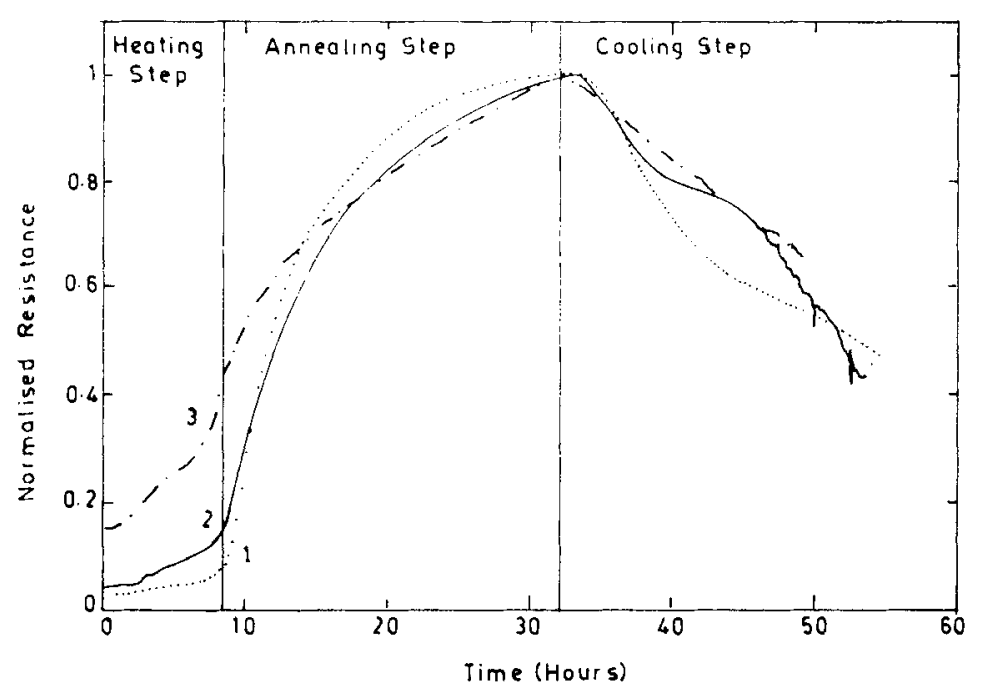

Figure 4. Resistivity as a function of time for YBCO samples undergoing heat treatment as discussed in text. Curves 1,2 and 3 correspond to annealing temperatures $\mathrm{TA}=453^{\circ} \mathrm{C}, 433^{\circ} \mathrm{C}$ and $383^{\circ} \mathrm{C}$ respectively. 
In the HTXRD studies carried out on a different set of samples, during the heating step there is a continuous expansion of the lattice. The lattice parameters corrected for thermal expansion can be plotted as a function of time covering the three thermal treatment regimes. It is illustrative to discuss a specific case with $T_{\mathrm{A}}=410^{\circ} \mathrm{C}$. In this case there exists two distinct phases at the end of the cooling step, namely a tetragonal and an orthorhombic phase. The thermal expansion corrected (006) $h k l$ reflection of the ortho phase shows an increase in the ' $c$ ' lattice parameter from 11.72 to $11.745 \AA$, whereas the ' $b$ ' lattice parameter increases from 3.862 to $3.878 \AA$ and the ' $a$ ' lattice parameter decreases from 3.840 to $3.835 \AA$. In the absence of any intake of oxygen from outside, this could be possible only under two situations: (i) a redistribution of oxygen atoms in the basal plane within the grains and (ii) better ordering in the basal plane of the sample during cooling. Low temperature ac susceptibility measurements on $410^{\circ} \mathrm{C}$ annealed sample showed a $T_{\mathrm{c}}$ of $60 \mathrm{~K}$, which corresponds to the $\mathrm{O}_{\mathrm{II}}$ phase. The results imply the coexistence of $\mathrm{O}_{\mathrm{II}}+\mathrm{T}$ phases.

\section{Superconductivity in doped fullerenes}

Kroto et al (1986) in their experiments on mass spectrometry of laser ablated graphite discovered the existence of stable clusters of 60 carbon atoms, and in a remarkable display of ingenuity, from this single observation of mass number, proposed the elegant soccer ball structure for the molecule with the 60 carbon atoms at the vertices of a truncated icosahedron. Soon carbon clusters with various other mass numbers were identified with $\mathrm{C}_{70}$ being next in predominance to $\mathrm{C}_{60}$ and the clusters came to be known by the general name of fullerenes. In 1990 Kratschmer et al (1990) published a method for the synthesis of fullerenes in macroscopic quantities, which gave rise to extensive studies of the $C_{60}$ molecule per se and also the structural, vibrational and electronic properties of its solid phases and doped derivatives. In one such study, metallicity and superconductivity $\left(T_{\mathrm{c}}=18 \mathrm{~K}\right)$ was found when potassium was intercalated in $\mathrm{C}_{60}$ (Hebard et al 1991). Further studies showed $\mathrm{Rb}_{3} \mathrm{C}_{60}$ to be superconducting at $29 \mathrm{~K}$ and $\mathrm{Rb}_{2} \mathrm{CsC}_{60}$ at $31.3 \mathrm{~K}$, the highest reported so far. Solid $\mathrm{C}_{60}$ intercalated with alkali metals exhibits a rich phase diagram. The $A_{3} C_{60}$ phase, which is the superconducting phase, has also the fcc structure with the alkali atoms at the tetrahedral and octahedral interstices.

The solid $\mathrm{C}_{60}$ has an fec structure with a lattice parameter of $14.17 \mathrm{~A}$ and exhibits interesting structural transitions (Devadasan et al 1994) on lowering the temperature, which are associated with the orientational ordering of the molecules. $\mathrm{C}_{60}$ is in itself insulating with a band gap estimated between 1 and $2.3 \mathrm{eV}$, between the five-fold degenerate highest occupied molecular orbital (HOMO) and the three-fold degenerate lowest unoccupied molecular orbital (LUMO). The alkali metal atoms donate electrons to $\mathrm{C}_{60}$, resulting in the filling of the LUMO band in proportion to the number of intercalated $A$ atoms. Thus for $A_{3} C_{60}$, the band is half filled and the density of states is maximum and the conductivity is also maximum. For $\mathrm{A}_{6} \mathrm{C}_{60}$ the band is completely filled, the structure changes to bcc to accommodate the additional $\mathrm{A}$ atoms and the material becomes insulating again. There is also a bet variant with the composition $\mathrm{A}_{4} \mathrm{C}_{60}$, which is also insulating. Raman spectra show evidence of charge transfer to the $\mathrm{C}_{60}$ molecules. As the concentration of dopant molecules increases, the molecule oscillates at a lower frequency.

One of the main interests in the study of superconductivity in this class of materials is to identify the mechanism of pairing. Both the standard BCS electron-phonon mechanism and another that emphasises the importance of electron correlations are candidates. 


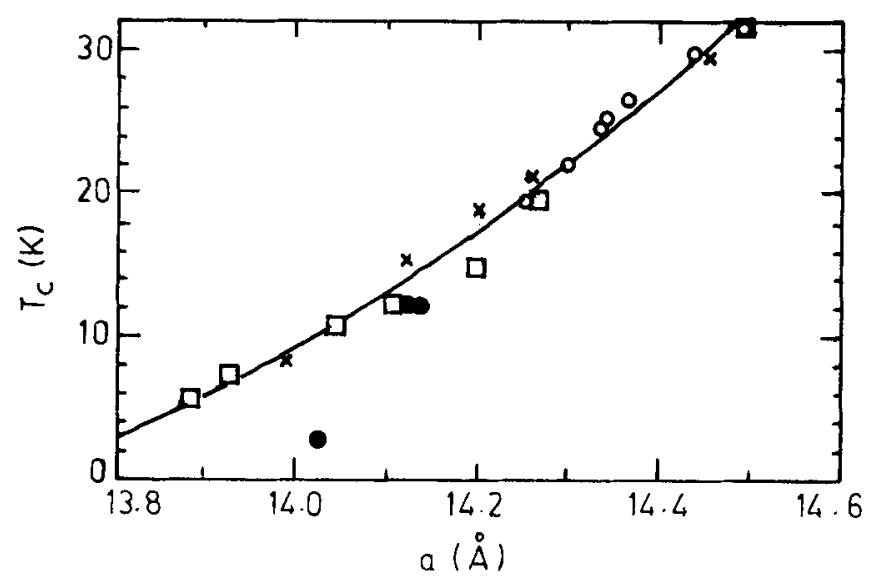

Figure 5. Superconducting transition temperature vs lattice parameter. Open squares and crosses represent $\mathrm{K}_{3} \mathrm{C}_{60}$ and $\mathrm{Rb}_{3} \mathrm{C}_{60}$ data from pressure experiments. Circles are ambient pressure data for various alkali doped systems.

Table 2. Superconducting parameters in $\mathrm{A}_{3} \mathrm{C}_{60}$.

\begin{tabular}{lcc}
\hline Parameter & $\mathrm{A}=\mathrm{Rb}$ & $\mathrm{A}=\mathrm{K}$ \\
\hline$T_{\mathrm{c}}(\mathrm{K})$ & $29 \cdot 6$ & $19 \cdot 3$ \\
$\left(\mathrm{~d} T_{\mathrm{c}} / \mathrm{d} p\right)(\mathrm{K} / \mathrm{GPa})$ & $-0 \cdot 97$ & $-0 \cdot 78$ \\
$H_{\mathrm{c} 1}(\mathrm{mT})$ & 26 & 13 \\
$H_{\mathrm{c} 2}(\mathrm{~T})$ & 34 & 26 \\
$J_{\mathrm{c}}\left(10^{6} \mathrm{~A} / \mathrm{cm}^{2}\right)$ & $1 \cdot 5$ & $0 \cdot 12$ \\
$\xi(\mathrm{nm})$ & $3 \cdot 0$ & $3 \cdot 5$ \\
$\lambda(\mathrm{nm})$ & 440 & 480 \\
$\kappa$ & 84 & 92 \\
\hline
\end{tabular}

One of the important correlations that has been established from the studies on alkali doped fullerenes is the variation of $T_{\mathrm{c}}$ with lattice parameter of the superconducting fcc phase (Katsumi et al 1993). This has been obtained from the studies on various alkali doped $\left(\mathrm{A}_{1} \mathrm{~A}_{2}\right)_{3} \mathrm{C}_{60}\left(\mathrm{~A}_{1}, \mathrm{~A}_{2}: \mathrm{K}, \mathrm{Rb}, \mathrm{Cs}\right)$ systems, and from the pressure dependence of $T_{\mathrm{c}}$ and lattice parameter in $\mathrm{K}_{3} \mathrm{C}_{60}$ and $\mathrm{Rb}_{3} \mathrm{C}_{60}$ (Schirber et al 1991) and plotted in figure 5. $T_{c}$ is dependent only on the lattice parameter and not on the identity of the alkali atom. The increase in $T_{\mathrm{c}}$ can be explained in terms of the increase in the density of states at the Fermi level. The variation seen in figure 5 is qualitatively consistent with the theoretical models based on either electron correlations or electron-phonon interactions. But detailed quantitative agreement has been shown within the framework of electron-phonon model. Properties of $\mathrm{K}_{3} \mathrm{C}_{60}$ and $\mathrm{Rb}_{3} \mathrm{C}_{60}$ are shown in table 2 .

\section{Quarternary borocarbide superconductors}

The pioneering discovery of weak superconductivity, in a sample thought to be $\mathrm{YNi}_{4} \mathrm{~B}$ (Mazumdar et al 1993), and the role of carbon contamination in the superconductivity of the material (Nagarajan et al 1994) led to the-new family of quarternary borocarbide 


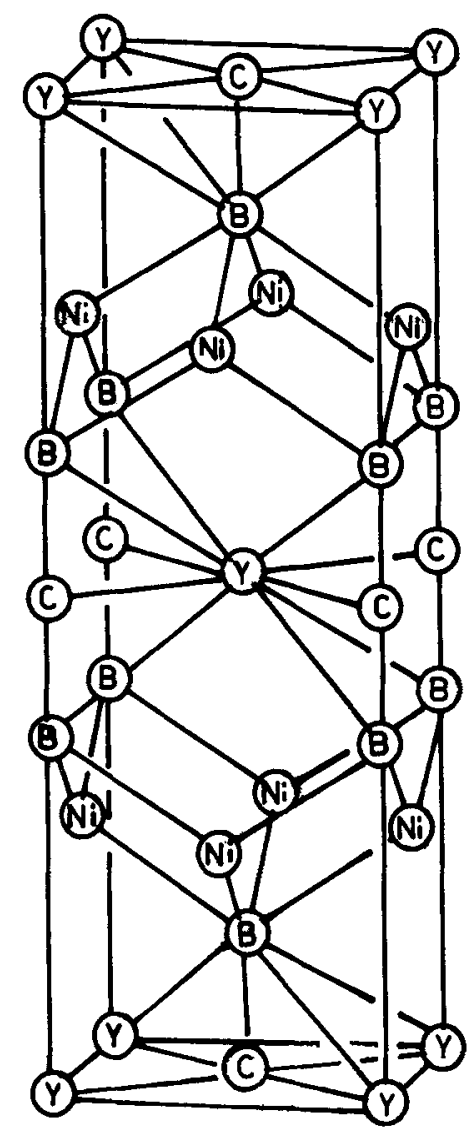

Figure 6. Crystal structure of $\mathrm{YNi}_{2} \mathrm{~B}_{2} \mathrm{C}$.

superconductors. Subsequent to this superconductivity was identified (Cava et al 1994) in $\mathrm{RNi}_{2} \mathrm{~B}_{2} \mathrm{C}\left(\mathrm{R}=\mathrm{Y}, \mathrm{Lu}, \mathrm{Tm}, \mathrm{Er}\right.$ and Ho with $T_{\mathrm{c}}$ of $15.6 \mathrm{~K}, 16.6 \mathrm{~K}, 11 \mathrm{~K}, 10.5 \mathrm{~K}$ and $8 \mathrm{~K}$ respectively). These intermetallic borocarbide samples are synthesized by arc melting the constituents followed by annealing at $1050^{\circ} \mathrm{C}$. The borocarbides belong to the space group $I 4 / \mathrm{mmm}$. The widely studied $\mathrm{YNi}_{2} \mathrm{~B}_{2} \mathrm{C}$ has the following lattice parameter: $a=3.526 \AA$ and $c=10 \cdot 543 \AA$.

The structure of $\mathrm{YNi}_{2} \mathrm{~B}_{2} \mathrm{C}$ is shown in figure 6 . This is a derivative of the well known $\mathrm{ThCr}_{2} \mathrm{Si}_{2}$ tetragonal structure with the $\mathrm{C}$ atoms inserted in the $\mathrm{Y}$ planes (Siegrist et al 1994). $\mathrm{YNi}_{2} \mathrm{~B}_{2}$ does not form and the introduction of $\mathrm{C}$ atoms between $\mathrm{B}$ atoms along the $\mathrm{c}$ axis increases the ' $c$ ' parameter thereby sustaining the structure. Positron studies (Sundar et al 1996) have inferred the existence of ordered carbon vacancies from life time rreasurements and positron density calculations.

The systematic decrease in $T_{\mathrm{c}}$ in $\mathrm{RNi}_{2} \mathrm{~B}_{2} \mathrm{C}$ with the increase in $\mathrm{RE}$ magnetic moment is indicative of a weak coupling between RE magnetic moment and the conduction electrons. Substitution with $\mathrm{Fe}$ and $\mathrm{Co}$ for $\mathrm{Ni}$ also depresses $T_{\mathrm{c}}$. This may mean that the $3 \mathrm{~d}$ electrons have a role to play in the superconductivity of this system. Band structure calculations show the existence of a peak at the Fermi energy. While it is true that the peak is contributed by all $\mathrm{Y}, \mathrm{Ni}$, $\mathrm{B}$ and $\mathrm{C}$ bands. the dominant contribution has been identified to be due to $\mathrm{Ni}-3 \mathrm{~d}$ electrons. 
Among the several borocarbides, $\mathrm{HoNi}_{2} \mathrm{~B}_{2} \mathrm{C}, \mathrm{ErNi}_{2} \mathrm{~B}_{2} \mathrm{C}$ and $\mathrm{TmNi}_{2} \mathrm{~B}_{2} \mathrm{C}$ have magnetism coexisting with superconductivity. In a single crystal of $\mathrm{HoNi}_{2} \mathrm{~B}_{2} \mathrm{C}$ $\left(T_{\mathrm{c}}=8 \mathrm{~K}\right)$, neutron diffraction studies have shown the existence of an AFM state below $4.7 \mathrm{~K}$ and also the existence of an incommensurate modulated magnetic structure between 4.7 and $6 \mathrm{~K}$ (Nagarajan 1994 for a general review).

Upper critical field measurements in $\mathrm{YNi}_{2} \mathrm{~B}_{2} \mathrm{C}$ and $\mathrm{Y}-\mathrm{Pd}-\mathrm{B}-\mathrm{C}$ indicate a positive curvature as a function of $T_{\mathrm{c}}$, which is not universally observed in all superconductors (Kalavathi et al 1995). The observed $\mathrm{H}_{\mathrm{c} 2}-T$ variation has been analysed using the clean limit approach and a model based on the existence of Landau levels seems to explain the positive curvature in $\mathrm{YNi}_{2} \mathrm{~B}_{2} \mathrm{C}$. While there may be no difficulty in classifying the borocarbides as conventional superconductors (unlike HTSC) with phonon mediated pair interaction resulting in superconductivity, it is yet to be resolved as to what may be the nature of interactions among the RE moments, conduction electrons and the $3 \mathrm{~d}$ electrons of $\mathrm{Ni}$.

\section{Applications of superconductivity}

\subsection{International context-low temperature superconductors (LTSC)}

Broadly the superconducting technology has grown in two directions. The first comprises the high current applications where the loss less transport of electric current in superconductors is exploited mainly as an energy saving device in the realization of compact electromagnets for a variety of uses. Today magnetic resonance imaging (MRI) systems for clinical diagnostics represents the most widespread of all the uses of superconducting magnets. Several superconducting high gradient magnetic separation systems (S-HGMS) have been used for mineral ore processing, clay beatification and for water treatment. Superconducting magnetic energy storage (SMES) systems of capacity up to $30 \mathrm{MJ}$, and superconducting electric generators up to $300 \mathrm{MVA}$ have been built to demonstrate the feasibility and advantages. High energy particle accelerators and experimental fusion machines have been built and operated with superconducting magnets as essential constituents. Yet for all these applications the single common crucial input has been availability through development, stable and high current carrying conductor. Mostly the niobium-titanium alloys (see $\S 2$ ) and to a smaller extent $\mathrm{Nb}_{3} \mathrm{Sn}$ have been the staple conductor materials.

The other major direction comprises the low current applications and centres around the Josephson junctions and other quantum effects. Superconducting quantum interference devices (acronym SQUID) incorporating Josephson junctions have been configured as sensitive magnetometers with unparalleled sensitivity. Their potential have been demonstrated in geomagnetic exploration for mineral prospecting, biomagnetic clinical diagnostics and as magnetic anomaly detectors for non-destructive testing and also in other strategic applications. For all these applications the development of $\mathrm{Nb}-\mathrm{Al}_{2} \mathrm{O}_{3}-\mathrm{Nb}$ tunnel junctions through microfabrication has been the single common crucial input.

\subsection{International context - high temperature superconductors (HTSC)}

Due to the intrinsically poor mechanical and electronic properties of the HTSC, despite a decade of development, both types of crucial inputs viz. the high current conductors 
Table 3. Indian developments of components and systems using LTSC reflecting the current strengths in superconductivity applications.

\begin{tabular}{lll}
\hline Development & Major specification & Institutions \\
\hline
\end{tabular}

1. Core enabling technologies

(i) Nb-Ti wires Multifilamentary:

IGCAR, BARC

$J_{c} \sim 2200 \mathrm{~A} / \mathrm{mm}^{2}$ at

$(5 \mathrm{~T}, 4.2 \mathrm{~K})$

(ii) $\mathrm{Nb}-\mathrm{Al}_{2} \mathrm{O}_{3}-\mathrm{Nb}$ Josephson junctions

2. Superconducting magnets for research and applications

(i) $\mathrm{Nb}-\mathrm{Ti}$ solenoids

(ii) $\mathrm{Nb}$-Ti high homogeneity solenoids

(iii) Symmetric and asymmetric coils

(iv) $\mathrm{NbTi} / \mathrm{Nb}_{3} \mathrm{Sn}$ hybrid coils

(v) $\mathrm{Nb}$-Ti magnet for $100 \mathrm{MHz}$ NMR spectrometer

(vi) $\mathrm{Nb}$-Ti magnet for small bore MRI (under execution)

3. Superconducting high gradient magnetic separator (S-HGMS)

(a) Experimental S-HGMS for ore separation

(b) Experimental S-HGMS

(c) S-HGMS for processing Kaolinitic clay in the commercial sector (under execution)

4. (a) Superconducting generator (b) Superconducting generator (under execution)

5. Thin film DC SQUID

6. SQUID magnetometer (under execution)

7. Josephson series array Voltage standard upto 8 Tesla

upto 6 Tesla

upto 4 Tesla

11 Tesla

6th order coil; further

homogeneity by shim coils

Persistent current mode

$100 \mathrm{~mm}$ dia warm bore;

5 Tesla

warm bore $3.5 \mathrm{~T}$

warm bore dia $320 \mathrm{~mm}$

3.5 Tesla

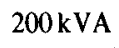

5 MVA

$\mathrm{Nb}-\mathrm{Al}_{2} \mathrm{O}_{3}-\mathrm{Nb}$

junction,

Flux noise: $25 \mu \phi_{0} / \sqrt{\mathrm{Hz}}$
IGCAR, BARC, NPL, CAT

IGCAR, BARC, NPL, CAT

BARC

NPL

NPL

CAT

BHEL and NPL

BARC

BHEL and EICL
6 Tesla

4-300 K

Assembled from a series array of 3000 Josephson junctions 
Table 4. Developments in India towards HTSC applications.

\begin{tabular}{lll}
\hline Development & Major specifications & Institutions \\
\hline $\begin{array}{l}\text { Bulk materials } \\
\text { targets and shapes }\end{array}$ & $\begin{array}{l}\text { Materials with optimized } \\
\text { properties developed in } \\
\text { laboratories }\end{array}$ & $\begin{array}{l}\text { Several; BARC, } \\
\text { IIT(B), IGCAR }\end{array}$ \\
$\begin{array}{l}\text { Melt textured YBCO suitable } \\
\text { for bearing applications }\end{array}$ & High $J_{\mathrm{c}}$ & DMRL \\
SQUIDs & $\begin{array}{l}\text { Thick film RF SQUID with } \\
\text { white noise } 2 \mathrm{pT} / \sqrt{\mathrm{Hz}} \text { at } 1 \mathrm{~Hz}\end{array}$ & NPL \\
BSCCO wire & $10^{4} \mathrm{~A} / \mathrm{cm}^{2}$ & RRL (T) \\
& $(0 \mathrm{~T}, 77 \mathrm{~K})$ & BHEL \\
\hline
\end{tabular}

and the tunnel junctions of the required reliability have not yet been realized, making the major applications of HTSC atleast a few years away. Notwithstanding the above difficulties, impressive developments have taken place towards some ingenuous applications of HTSC. Among them is the use of single component thin films patterned into microwave resonators and filters, exploiting the low surface resistance of the films at $77 \mathrm{~K}$ at $\mathrm{GHz}$ frequencies. SQUIDs involving different types of weak links and not necessarily tunnel junctions have been made. YBCO has been specially processed to carry high circulating $J_{c}$ and has been exploited for use in frictionless superconducting magnetic bearings. Bulk YBCO as well as $\mathrm{Bi} 2212$ have been developed as low thermal load high current leads for charging conventional (LTSC) superconducting magnets that allow operation of the magnets using portable closed cycle refrigerators rather than with liquid helium.

\subsection{Current Indian strengths in superconductivity applications}

It was mentioned in $\S 1.1$ that the high current carrying conductor and the Josephson tunnel junction are the two crucial primary requirements for all the major applications of superconductivity. In this sense a major strength is the availability through entirely indigenous developments, $\mathrm{Nb}-\mathrm{Ti}$ multifilamentary wire and $\mathrm{Nb}-\mathrm{Al}_{2} \mathrm{O}_{3}-\mathrm{Nb}$ junction which together can be regarded as the enabling technologies (Radhakrishnan 1994). Further a range of superconducting systems have been designed, developed and commissioned along with the development of the associated cryogenic technology. Table 3 depicts the major developments that have taken place in the country in the realm of LTSC. Table 4 shows the developments in HTSC applications.

\section{Acknowledgements}

We acknowledge the stimulating atmosphere provided by colleagues pursuing superconductivity research in IGCAR. We also acknowledge the support of Dr Placid Rodriguez, Director IGCAR and Dr Baldev Raj, Director, Metallurgy and Materials Group for the growth of this field in IGCAR. 


\section{References}

Awadhesh M, Vaidhyanathan L S, Hariharan Y, Janawadkar M P and Radhakrishnan T S 1996 Cryogenics 36937

Baillif R, Dunand A, Muller J and Yvon K 1981 Phys. Rev. Lett. 47672

Baskaran R, Hariharan Y, Janawadkar M P and Radhakrishnan T S 1988 Physica C158 406

Beasley M R 1995 IEEE Trans. Appl. Supercond. 5141

Bednorz J-G and Muller K A 1986 Z. Phys. B64 189

Cava R J et al 1994 Nature 367252

Collings E W 1983 in Source book of titanium alloy superconductivity (New York: Plenum)

Devadasan K V et al 1994 Solid State Commun. 92715

Fisher O 1991 Extended abstract of the int. workshop on chevrel phase superconductors (Switzerland: Univ. of Geneva)

Geetha B, Srinivasan R, Sankaranarayanan V, Rangarajan G, Janaki R and Subba Rao G V 1984 Proc. 17th int. conf. on low temperature physics (eds) U Eckern, A Schmid, W Weber and H Wuhl (Amsterdam: North Holland) p. 343

Goldacker W, Mirrglia S, Hariharan Y, Wolf T and Flukiger R 1988 Advances in cryogenic engineering materials (eds) A F Clark and R P Reed (New York: Plenum Press) 34 p. 655

Hariharan Y, Janawadkar M P, Radhakrishnan T S, Terrance A L E, Dixit G A and Raghunathan V S 1986a Pramana-J. Phys. 26513

Hariharan Y, Janawadkar M P and Radhakrishnan T S 1986b Solid State Commun. 59127

Hariharan Y, Janawadkar M P, Sastry V S and Radhakrishnan T S 1988 Rev. Solid State Sci. 2181

Hebard A F, Rosseinsky M J, Haddon R C, Glarum S H, Palstra T T M, Ramirez A P and Kortan A R 1991 Nature 350600

Hirrien M 1986 Thesis, University of Rennes, France

Janawadkar M P, Sankara Sastry V, Hariharan Y and Radhakrishnan T S 1984 J. Low Temp. Phys. 54411

Jorgensen J D and Hinks D G 1985 Solid State Commun. 53289

Kalavathi $\mathrm{S}$ et al 1995 in Int. symp. on strongly correlated electron systems, Goa (to appear in Physica B)

Kanithi H C, Valaris P, Motowidto L R and Zeitlin B A 1992 in Advances in cryogenic engineering materials (eds) F R Fickett and R P Reed (Plenum) B38 p. 675

Katsumi T, Ichiro H, Ebbesen T W, Mizuki J-I and Tsai J-S 1993 J. Phys. Chem. Solids 541645

Kratschmer W, Lamb L D, Fostiropoulos K and Huffman D R 1990 Nature 347354

Kroto H W, Heath J R, O'Brien S C, Curl R F and Smalley R E 1985 Nature 312162

Locquet J P and Machler E 1994 MRS Bull. 1939

Mazumdar Cet al 1993 Solid State Commun. 87413

Nagarajan R 1994 Indian J. Pure Appl. Phys. 334753

Nagarajan R et al 1994 Phys. Rev. Lett. 72274

Obst B, Pattnayak D and Hochstuhl P 1980 J. Low Temp. Phys. 4158

Radhakrishnan T S 1987 Pramana-J. Phys. 28555

Radhakrishnan T S 1994 Curr. Sci. 67891

Radhakrishnan T S, Hariharan Y and Janawadkar M P 1989a Metal Mater. \& Process. 21977

Radhakrishnan T S et al 1989b Pramana-J. Phys. 321705

Rao G V N, Sastry V S, Hariharan Y and Radhakrishnan T S 1996 (to be published)

Sankara Sastry V, Hariharan Y, Janawadkar M P and Radhakrishnan T S 1988 Rev. Solid State Sci. 2195

Schirber J E, Overmyer D L, Wang H H, Williams J M, Carlson K D, Kini A M, Welp U and Kwok W-K 1991 Physica $\mathbf{C 1 7 8} 137$

Siegrist T, Zandbergen H W, Cava R J, Krajeswski J J and Peck Jr W F 1994 Nature 367254

Sood A K, Sankaran K, Hariharan Y, Vijayalakshmi S, Sankara Sastry V, Kalavathi S and Janaki J 1988 Pramana-J. Phys. 31389

Sundar C S, Bharathi A, Hariharan Y, Radhakrishnan T S, Hossain Z, Nagarajan R, Gupta L C and Vijayaraghavan R 1996 Phys. Rev. B53 R2971

Sundaram C V and Radhakrishnan T S 1989 Bull. Mater. Sci. 12225

Yao Y S, Guertin R P, Hinks D G, Jorgensen J and Kapone D W 1988 Phys. Rev. B37 5032

Zeldov E, Majer D, Konczykowski M, Gesjkenbein V B, Vinokur V M and Shtrikman H 1995 Nature 375 373 